\title{
Antecedente de agresión sexual y su asociación con conductas de riesgo en adolescentes consultantes en un centro de atención en salud sexual y reproductiva
}

\author{
Electra González A. ${ }^{1 a}$, Paulina Troncoso E. ${ }^{1}$ Temístocles Molina G. ${ }^{1 b}$, Vania Martínez N. $^{1}$ \\ ${ }^{1}$ Centro de Medicina Reproductiva y Desarrollo Integral del Adolescente (CEMERA), Facultad de Medicina, Universidad \\ de Chile.
}

${ }^{\mathrm{a}}$ Asistente Social, MSc in Population Research. ${ }^{\mathrm{b}}$ Bioestadístico, Magíster en Bioestadística.

\begin{abstract}
RESUMEN
Antecedentes: La mayoría de los programas de salud reproductiva en Chile operan bajo la premisa de que todas las mujeres y en especial las adolescentes están o han estado comprometidas en relaciones sexuales consensuales, y no consideran que muchas de ellas pueden ser sobrevivientes de agresión sexual y pueden presentar necesidades diferentes en salud sexual y reproductiva. Objetivo: Examinar cuáles conductas de riesgo y variables sociodemográficas y familiares están asociadas al antecedente de agresión sexual en adolescentes consultantes en un centro de atención en salud sexual y reproductiva. Método: Estudio analítico de corte transversal. La información fue recolectada en la primera consulta a 3.064 adolescentes mujeres entre 12 y 19 años, atendidas en un centro de salud sexual y reproductiva en el período 2003-2010. Se usó regresión logística para identificar los factores asociados a la agresión sexual. Resultados: Un 22,7\% reportó antecedente de agresión sexual. Las conductas de riesgo asociadas al antecedente de agresión sexual fueron: inicio de la actividad sexual bajo coerción o bajo efectos del alcohol, mayor número de relaciones afectivas y parejas sexuales, menor edad al inicio de la actividad sexual, menor promedio de notas, mayor consumo de cigarrillo, de alcohol y drogas, y menor edad al inicio del consumo de drogas. Conclusión: El antecedente de agresión sexual deja a las mujeres expuestas a riesgos en la salud sexual y reproductiva, por lo que los equipos de salud debieran ser capaces de detectar estas problemáticas para poder intervenir en forma adecuada y eficaz.
\end{abstract}

\section{PALABRAS CLAVE: Agresión sexual, adolescencia, variables sociales, conductas de riesgo, pro- gramas de salud reproductiva}

\section{SUMMARY}

Background: Most of reproductive health programs in Chile operate under the premise that women and adolescent girls in particular, are or have been engaged in consensual sex and do not consider that many of them may be survivors of sexual abuse, and may have different needs in sexual and reproductive health. Objective: Determine which risk behaviors and familial variables are associated with sexual aggression history in adolescents consulting at sexual and reproductive health center. Method: Cross-sectional analytical study. The information was gathered from a structured interview applied in the first consultation to 3,064 adolescent girls between 12 and 19 years, who attended a sexual and reproductive health centre for the period 2003-2010. Logistic regression was used to identify factors associated with sexual assault. Results: A 22.7\% reported sexual assault history. Risk behaviors associated with sexual aggression were: onset of sexual activity forced or under influence of alcohol, as many relationships and sexual partners, younger age 
at onset of sexual activity, lower grade point average, cigarette smoking, alcohol and drug use and younger age at onset of drug use. Conclusion: A history of sexual aggression leaves women at risk for sexual and reproductive health, so health teams should be able to detect these problems in order to intervene appropriately and effectively.

\section{KEY WORDS: Sexual assault, teenagers, social variables, risk behaviors,} reproductive health programs

\section{INTRODUCCIÓN}

En Chile, hasta ahora, la mayoría de los programas de salud sexual y reproductiva operan bajo la premisa de que todas las mujeres y en especial las mujeres adolescentes están o han estado comprometidas en relaciones sexuales consensuales, sin consideran que muchas de ellas pueden ser víctimas de abuso sexual y presentar necesidades diferentes en salud sexual y reproductiva. La agresión sexual es un evento traumático que afecta, de manera transversal, a las víctimas de todos los niveles socioeconómicos y donde el perpetrador generalmente es un hombre y las víctimas son mujeres en la etapa más vulnerable como es la niñez y la adolescencia (1-4).

En Latinoamérica se reporta que en un $12 \%$ el inicio sexual es en menores de 14 años, y entre un 15 a un $30 \%$ el inicio sexual es bajo coerción (5). En Chile, durante el año 2010 se reportaron 6.956 denuncias por agresiones sexuales, de éstas 5.444 afectaron a mujeres y 1.512 a hombres, concentrándose en la Región Metropolitana el mayor número de denuncias, con 1.139 por violación y 2.311 por abuso sexual; las edades más vulnerables fueron entre 5 y 14 años (6). Un estudio previo mostró que el $22,7 \%$ de adolescentes consultantes en Cemera reportaron agresiones sexual y el promedio de edad en que ocurrió este evento fue 10,1 años (7).

En el marco de la Conferencia del Cairo de 1994, Chile se comprometió, como país firmante, a proteger, garantizar y promover el ejercicio de derechos en material de salud sexual y reproductiva, y a impulsar políticas públicas destinadas a eliminar todas las formas de violencia contra las mujeres, adolescentes y niñas. La salud sexual incorpora el normal desarrollo y la satisfacción de necesidades humanas básicas, como contacto, intimidad, expresión de emociones y placer; esta dimensión del desarrollo se quiebra con la agresión sexual, al distorsionarse la percepción de la propia sexualidad (8).

Si bien se describe que la mayoría de los traumas son en la esfera psicológica (9-12), es importante considerar que las mujeres que han sufrido agresión sexual tienen además necesidades en salud, y en especial en salud sexual y reproductiva diferentes a aquellas que no han sido víctimas de agresión sexual, y los servicios de salud deberían estar acordes con estas necesidades.

Los efectos de la agresión sexual infantil en la salud sexual y reproductiva de las mujeres que la han sufrido, son múltiples y afectarán su vida sexual, social y familiar, a corto y a largo plazo. Las víctimas de agresión sexual, en particular, las que han sufrido incesto, presentan serias disfunciones en el área de su sexualidad como: mayores niveles de ansiedad en la vida sexual, culpa por su vida sexual y una gran insatisfacción en sus relaciones sexuales cuando se comparan con mujeres sin antecedente de abuso sexual infantil $(10,13)$. Inhabilidad para relajarse y disfrutar la actividad sexual, abstinencia sexual y otras disfunciones sexuales están presentes en estas mujeres. Ellas experimentan menos orgasmos, y obtienen una baja satisfacción de sus parejas sexuales, sintiéndose menos satisfechas de la calidad de las relaciones que establecen con los varones $(14,15)$.

Por otro lado, se describe que reportan más comportamientos sexuales riesgosos en esta esfera como inicio sexual más temprano, actividad sexual no protegida, más parejas sexuales (16-19), involucramiento en comercio sexual y/o prostitución (20-23), aumento del riesgo de infecciones de transmisión sexual (ITS), entre ellas el VIH-SIDA $(10,24-26)$, y el embarazo producto de violación son otras de las graves consecuencias reportadas de las agresiones sexuales (27-30).

La literatura muestra también una fuerte asociación entre agresión sexual y otros comportamientos de riesgo en la adolescencia como abuso de alcohol y drogas comparadas con aquellas que no sufrieron esta experiencia $(31,32)$. Sin embargo, también se ha encontrado que hay factores familiares asociados a la agresión sexual tales como desventajas sociales, malas relaciones entre padres e hijos, inestabilidad familiar, no crianza por ambos padres, entre otros (33). Finalmente otro impacto que es importante considerar es la alta vulnerabilidad de estas mujeres para ser víctimas nuevamente de agresión sexual (34). No hay estudios realizados en Chile que muestren la asociación entre antecedente de agresión sexual y posteriores conductas de riesgo en adolescentes consultantes en salud sexual y reproductiva.

El objetivo de este estudio fue determinar los 
comportamientos de riesgo y los factores sociodemográficos y familiares que están asociados al antecedente de agresión sexual, en adolescentes consultantes en un centro de atención de salud sexual y reproductiva.

\section{PACIENTES Y MÉTODO}

Diseño de estudio transversal analítico, que analizó tanto comportamientos de riesgo como variables sociodemográficas y familiares previamente identificados en la literatura internacional, como asociados al antecedente de agresión sexual en una población de adolescentes mujeres. La población del estudio fueron adolescentes mujeres que consultaron en un centro de salud sexual y reproductiva (CEMERA) entre enero de 2003 y diciembre de 2010. CEMERA es el primer y más importante centro de salud sexual y reproductiva situado en la Región Metropolitana, que provee atención integral y confidencial para adolescentes de nivel socioeconómico medio y bajo por parte de un equipo multiprofesional altamente especializados en la atención de adolescentes.

Los datos se obtuvieron desde la ficha clínica que aplicó la investigadora principal al ingreso de cada adolescente. A todas las adolescentes se les solicitó permiso explícito para utilizar los datos, asegurando el resguardo de la confidencialidad y anonimato de los datos. Lo anterior fue formalizado mediante firma del consentimiento informado especialmente diseñado para estos efectos. No se condicionó la atención a la participación en el estudio. Los datos fueron traspasados a una base de datos omitiendo nombres, número de ficha, domicilio, rol único nacional (RUN), o cualquier antecedente que pudieran identificar a las adolescentes del estudio. El acceso a la base de datos solo fue permitido a los investigadores que participaron en la investigación.

A todas las adolescentes que reportaron antecedente de abuso sexual, se les ofreció atención en salud mental en CEMERA sin costo, como también apoyo y orientación en el proceso de denuncia cuando esta no había sido realizada. El estudio fue aprobado por el Comité de Ética en Seres Humanos de la Facultad de Medicina de la Universidad de Chile.

Se definió agresión sexual de acuerdo a la definición del Código Penal de Chile (35). Se compararon dos grupos: adolescentes que reportaron haber tenido cualquier agresión sexual (casos) con aquellas que no habían tenido agresión sexual (controles).

Los comportamientos de riesgo analizados fueron: edad de inicio de la actividad sexual voluntaria, actividad sexual no protegida (no uso de anticonceptivo), número de relaciones afectivas, número de parejas sexuales, tipo de relación con la pareja que inician actividad sexual, razones del inicio sexual, tiempo entre inicio de la relación de pareja e inicio sexual, consumo de tabaco, edad de inicio de consumo de tabaco, consumo de alcohol, edad de inicio de consumo de alcohol, consumo de drogas, edad de inicio de consumo de drogas, rendimiento escolar (promedio de notas del último año cursado), repitencia escolar (sí/no).

Las variables sociodemográficas y familiares analizadas fueron: edad a la entrevista, actividad, escolaridad, participación social, afiliación religiosa, filiación (vínculo legal entre padres-hijos, matrimonial y no matrimonial), tamaño familia (número de hijos), orden de nacimiento, responsable de la supervisión, tipo de permiso, tipo de sanción al quebrantamiento de las normas familiares (grave, moderada y sin sanción), con quién conversa temas de sexualidad, escolaridad paterna y materna (años de escolaridad cursados), APGAR familiar, calidad de la relación con los padres (definida por auto reporte como buena, regular, mala), comunicación familiar (definida por auto reporte como buena, regular, mala)

Los resultados para las variables medidas en escala numérica, fueron expresados como valores promedios y porcentajes para las medidas en escala nominal. Se realizaron análisis uni y bivariado aplicándose las pruebas estadísticas Test Chi2 y Test de Mann-Whitney. Un primer análisis bivariado consistió en analizar los comportamientos de riesgo asociados a la agresión sexual (edad del inicio de la actividad sexual, número de parejas sexuales, actividad sexual no protegida, consumo de alcohol, tabaco y drogas). Luego se analizó las variables familiares asociadas a la agresión sexual en ambos grupos. Posteriormente se aplicó el método de selección de variables Backward a dos modelos de regresión logística múltiple, uno para identificar los comportamientos de riesgo asociados a la agresión sexual y otro para identificar aquellas variables familiares asociadas a la agresión sexual. Para este análisis se incluyeron en los dos modelos variables que en el análisis bivariado tuvieron un valor $\mathrm{p} \leq 0,20$ (36). Para evaluar la bondad del ajuste de los modelos se utilizó la prueba de Hosmer y Lemeshow (36).

\section{RESULTADOS}

El estudio comprende a 3.064 adolescentes mujeres entre 12 y 19 años. Un 22,7\% reportó antecedente de agresión sexual. La edad promedio a la entrevista fue de 15,8 años, edad promedio a la agresión sexual fue 10,1 años, el $98,6 \%$ son estudiantes con un promedio de 10,3 años de escolaridad.

El análisis bivariado mostró que los comportamientos de riesgo asociados al antecedente de agresión sexual fueron: mayor número de parejas afectivas, mayor número de parejas sexuales, inicio sexual fue con pareja ocasional, o bajo coerción o bajo efectos del alcohol, consumo de 
tabaco, consumo de alcohol, consumo de drogas (Tabla I). Se asoció a la agresión sexual: la menor edad de inicio sexual, el menor tiempo de relación de pareja al inicio de la actividad sexual, la menor edad al inicio del consumo de drogas (Tabla II). Actividad sexual no protegida no fue asociada al antecedente de agresión sexual.

Las variables sociodemográficas y familiares categóricas asociadas al antecedente de agresión sexual fueron: no estudia, repitencia escolar, sin religión, filiación no matrimonial, mayor número de hermanos, supervisión por otros, permisos sin restricción durante la semana, permisos restringidos los fines de semana, sanción grave al quebrantamiento de las normas familiares, no conversa con nadie los temas de sexualidad, mala comunicación familiar, mala relación con los padres, que el padre sea trabajador no calificado y menor escolaridad, menor promedio de notas de la adolescente, y menor escolaridad de la madre dentro de las variables continuas (Tabla III).

Tabla I

CONDUCTAS DE RIESGO PARA ADOLESCENTES CON Y SIN ANTECEDENTE DE AGRESIÓN SEXUAL

\begin{tabular}{|c|c|c|c|}
\hline Variables y categorías & $\begin{array}{c}\text { Con agresión } \\
(\%)\end{array}$ & $\begin{array}{c}\text { Sin agresión } \\
(\%)\end{array}$ & Valor $p$ \\
\hline \multicolumn{4}{|c|}{$\mathrm{N}^{\circ}$ de relaciones románticas: } \\
\hline 0 & 3,3 & 7,6 & 0,000 \\
\hline 1 & 19,3 & 21,6 & \\
\hline 2 & 28,5 & 30,1 & \\
\hline 3 & 24,7 & 21,3 & \\
\hline $4 y+$ & 24,2 & 19,4 & \\
\hline \multicolumn{4}{|l|}{$\mathrm{N}^{\circ}$ de parejas sexuales: } \\
\hline 0 & 17,5 & 23,3 & \\
\hline 1 & 55,0 & 57,0 & \\
\hline 2 & 16,2 & 14,7 & \\
\hline $3 y+$ & 11,3 & 5,0 & 0,000 \\
\hline \multicolumn{4}{|c|}{ Tipo de pareja al inicio de actividad sexual: } \\
\hline Pololo $^{1}$ & 85,8 & 91,1 & \\
\hline Andante $^{2}$ & 7,9 & 5,2 & \\
\hline Ocasional & 6,3 & 3,7 & 0,001 \\
\hline \multicolumn{4}{|c|}{ Razón inicio actividad sexual: } \\
\hline Amor/otras & 95,4 & 97,8 & \\
\hline Coerción/alcohol & 4,6 & 2,2 & 0,004 \\
\hline \multicolumn{4}{|l|}{ Consumo de tabaco: } \\
\hline $\mathrm{Si}$ & 70,4 & 62,4 & 0,004 \\
\hline No & 29,6 & 37,6 & \\
\hline \multicolumn{4}{|l|}{ Consumo de alcohol: } \\
\hline $\mathrm{Si}$ & 71,7 & 63,8 & 0,003 \\
\hline No & 28,3 & 36,2 & \\
\hline \multicolumn{4}{|l|}{ Consumo de droga: } \\
\hline $\mathrm{Si}$ & 41,1 & 32,4 & 0,001 \\
\hline No & 58,9 & 67,6 & \\
\hline
\end{tabular}

${ }^{1}$ Pololo: chilenismo que significa una relación de pareja sin un compromiso mayor. ${ }^{2}$ Andante: chilenismo que significa una relación de pareja sin compromiso 
Tabla II

VARIABLES PERSONALES Y FAMILIARES Y COMPORTAMIENTOS DE RIESGO PARA ADOLESCENTES CON Y SIN ANTECEDENTE DE AGRESIÓN SEXUAL (MEDIANAS)

\begin{tabular}{|c|c|c|c|c|c|}
\hline \multirow[t]{2}{*}{ Variables } & \multicolumn{2}{|c|}{ Con agresión } & \multicolumn{2}{|c|}{ Sin agresión } & \multirow[t]{2}{*}{ Valor $p$} \\
\hline & $\mathrm{Me}$ & $\mathrm{RI}$ & $\mathrm{Me}$ & $\mathrm{RI}$ & \\
\hline Edad al momento de la entrevista (años) & 16 & 2 & 16 & 2 & 0,09 \\
\hline Escolaridad (años cursados) & 10 & 2 & 11 & 1 & 0,001 \\
\hline Promedio de notas (rango: $1-7$ ) & 5,4 & 0,8 & 5,5 & 0,7 & 0,001 \\
\hline Escolaridad padre (años cursados) & 11 & 4 & 12 & 3 & 0,10 \\
\hline Escolaridad madre (años cursados) & 11 & 4 & 12 & 3 & 0,000 \\
\hline Edad inicio actividad sexual (años) & 15 & 2 & 15 & 2 & 0,000 \\
\hline $\begin{array}{l}\text { Tiempo de relación afectiva al inicio de } \\
\text { actividad sexual (meses) }\end{array}$ & 5 & 7 & 6 & 8 & 0,033 \\
\hline Edad inicio consumo tabaco (años) & 14 & 3 & 14 & 2 & 0,094 \\
\hline Edad inicio consumo alcohol (años) & 14 & 2 & 15 & 1 & 0,06 \\
\hline Edad inicio consumo droga (años) & 14 & 1,5 & 15 & 1 & 0,013 \\
\hline
\end{tabular}

Me: mediana. RI: Percentil 75 - Percentil 25.

Tabla III

VARIABLES SOCIODEMOGRÁFICAS Y FAMILIARES PARA ADOLESCENTES CON Y SIN ANTECEDENTE DE AGRESIÓN SEXUAL

\begin{tabular}{|c|c|c|c|c|}
\hline Variables & Categorías & $\begin{array}{c}\text { Con agresión } \\
(\%)\end{array}$ & $\begin{array}{c}\text { Sin agresión } \\
(\%)\end{array}$ & Valor $p$ \\
\hline Actividad & $\begin{array}{l}\text { Estudia } \\
\text { No estudia }\end{array}$ & $\begin{array}{r}97,4 \\
2,6\end{array}$ & $\begin{array}{r}98,9 \\
1,1\end{array}$ & 0,003 \\
\hline Repitencia escolar & $\begin{array}{l}\mathrm{No} \\
\mathrm{Si}\end{array}$ & $\begin{array}{l}71,6 \\
28,4\end{array}$ & $\begin{array}{l}76,0 \\
24,0\end{array}$ & 0,019 \\
\hline Religión & $\begin{array}{l}\text { Católica } \\
\text { Otra religión } \\
\text { Sin religión }\end{array}$ & $\begin{array}{l}54,9 \\
23,8 \\
21,3\end{array}$ & $\begin{array}{l}62,4 \\
19,1 \\
18,5\end{array}$ & 0,002 \\
\hline Filiación de la hija & $\begin{array}{l}\text { Matrimonial } \\
\text { No matrimonial }\end{array}$ & $\begin{array}{l}66,2 \\
33,8\end{array}$ & $\begin{array}{l}73,2 \\
26,8\end{array}$ & 0,000 \\
\hline $\mathrm{N}^{\circ}$ de hermanos & $\begin{array}{l}0 \\
1 \\
2 \\
3 \\
4 y+\end{array}$ & $\begin{array}{r}1,0 \\
7,5 \\
25,8 \\
34,9 \\
30,8\end{array}$ & $\begin{array}{r}1,8 \\
7,6 \\
31,8 \\
31,6 \\
27,2\end{array}$ & 0,014 \\
\hline Orden dentro de los hermanos & $\begin{array}{l}1 \\
2 \\
3 \\
4 y+\end{array}$ & $\begin{array}{l}44,9 \\
25,0 \\
16,8 \\
13,3\end{array}$ & $\begin{array}{l}44,0 \\
28,8 \\
16,0 \\
11,2\end{array}$ & 0,177 \\
\hline Supervisión & $\begin{array}{l}\text { Ambos padres } \\
\text { Padre } \\
\text { Madre } \\
\text { Otros }\end{array}$ & $\begin{array}{r}30,5 \\
8,6 \\
46,4 \\
14,5\end{array}$ & $\begin{array}{r}35,7 \\
10,0 \\
46,9 \\
7,4\end{array}$ & 0,000 \\
\hline
\end{tabular}


CONTINUACION TABLA III

\begin{tabular}{|c|c|c|c|c|}
\hline Variables & Categorías & $\begin{array}{c}\text { Con agresión } \\
(\%)\end{array}$ & $\begin{array}{c}\text { Sin agresión } \\
(\%)\end{array}$ & Valor $p$ \\
\hline Tipo de permisos semana & $\begin{array}{l}\text { Muy restringido } \\
\text { Restringido } \\
\text { Sin restricción }\end{array}$ & $\begin{array}{r}68,4 \\
27,6 \\
4,0\end{array}$ & $\begin{array}{r}71,7 \\
26,6 \\
1,7\end{array}$ & $\begin{array}{l}0,001 \\
0,000\end{array}$ \\
\hline Tipo de permisos fin semana & $\begin{array}{l}\text { Muy restringido } \\
\text { Restringido } \\
\text { Sin restricción }\end{array}$ & $\begin{array}{l}17,0 \\
29,3 \\
53,7\end{array}$ & $\begin{array}{l}13,3 \\
36,9 \\
49,8\end{array}$ & 0,000 \\
\hline $\begin{array}{l}\text { Sanción al quebrantamiento de } \\
\text { las normas familiares }\end{array}$ & $\begin{array}{l}\text { Grave } \\
\text { Moderado } \\
\text { Sin sanción }\end{array}$ & $\begin{array}{r}7,1 \\
68,2 \\
24,7\end{array}$ & $\begin{array}{r}3,0 \\
71,7 \\
25,3\end{array}$ & \\
\hline Con quien conversa de sexualidad & $\begin{array}{l}\text { Madre/padre } \\
\text { Familiares/amigos/ } \\
\text { pololo/otros } \\
\text { Nadie }\end{array}$ & $\begin{array}{r}28,2 \\
65,7 \\
6,1\end{array}$ & $\begin{array}{r}33,4 \\
63,3 \\
3,3\end{array}$ & 0,001 \\
\hline Comunicación familiar & $\begin{array}{l}\text { Buena } \\
\text { Regular } \\
\text { Mala }\end{array}$ & $\begin{array}{l}42,1 \\
32,2 \\
25,7\end{array}$ & $\begin{array}{l}55,4 \\
29,0 \\
15,6\end{array}$ & 0,000 \\
\hline Actividad padre & $\begin{array}{l}\text { Profesional } \\
\text { Empresario } \\
\text { Trabajador calificado } \\
\text { Trabajador no calificado }\end{array}$ & $\begin{array}{r}4,1 \\
1,3 \\
65,4 \\
29,2\end{array}$ & $\begin{array}{r}5,8 \\
2,0 \\
70,0 \\
22,2\end{array}$ & 0,003 \\
\hline Relaciones familiares & $\begin{array}{l}\text { Buena } \\
\text { Regular } \\
\text { Mala }\end{array}$ & $\begin{array}{l}38,9 \\
44,4 \\
16,7\end{array}$ & $\begin{array}{r}52,3 \\
40,1 \\
7,6\end{array}$ & 0,000 \\
\hline
\end{tabular}

En el modelo de regresión logística múltiple para los comportamientos de riesgo, resultaron significativas para las siguientes variables: por un año de aumento en el tiempo de relación de pareja al inicio de actividad sexual, la posibilidad de tener el antecedente disminuye un $2 \%$; por un año de aumento en la edad inicio actividad sexual, la posibilidad de tener el antecedente disminuye un 15\%; cuando la razón de inicio de actividad sexual es forzada o por alcohol, la posibilidad de la adolescente de tener el antecedente de agresión sexual aumenta 1,01 veces, controladas por las restantes variables. $\mathrm{La}$ calidad del ajuste resultó con un $\mathrm{p}=20$ (prueba de Hosmer y Lemeshow36) (Tabla IV).

En el modelo de regresión logística para las variables sociodemográficas y familiares (Tabla V), resultaron significativas las siguientes variables que aumentan la posibilidad de la adolescente de tener el antecedente de agresión sexual: relación familiar mala o regular, permiso en la semana sin restricción, permiso en fin de semana sin restricción, trabajo no calificado del padre, no conversa con nadie sobre sexualidad y supervisión en sexualidad por otras personas; mientras que a mayor escolaridad de la adolescente y de su madre reducen la proba- bilidad de tener el antecedente de agresión sexual. La calidad del ajuste según la Prueba de Hosmer y Lemeshow resultó con un $\mathrm{p}=0,78$ (36).

\section{DISCUSIÓN}

El objetivo de este estudio fue investigar los comportamientos de riesgo y los factores sociodemográficos y familiares asociados al antecedente de una agresión sexual en una población adolescente femenina que consulta por salud sexual y reproductiva, como también los factores sociodemográficos y familiares asociados a la agresión sexual.

Concordante con lo reportado en otros estudios (16-19), las adolescentes de este estudio que refirieron antecedente de agresión sexual presentaron comportamientos sexuales de riesgo tales como inicio sexual más temprano, mayor número de parejas sexuales, inicio sexual en un contexto de pareja ocasional, bajo coerción o bajo efectos del alcohol, como también menor tiempo entre el inicio de la relación de pareja y el inicio sexual. Sin embargo, contrario a lo reportado por otros estudios $(16,18)$, este estudio no logró mostrar asociación entre actividad sexual no protegida y agresión sexual. 
Tabla IV

\section{AJUSTE DE MODELO DE REGRESIÓN LOGÍSTICA PARA LAS VARIABLES COMPORTAMIENTOS} DE RIESGO Y ANTECEDENTE DE AGRESIÓN SEXUAL

\begin{tabular}{lccc}
\hline Variables & Valor p & OR & IC 95\% \\
\hline Tiempo de relación de pareja al inicio de actividad sexual (meses) & 0,013 & 0,98 & $(0,97-0,99)$ \\
Edad inicio actividad sexual (años) & 0,000 & 0,85 & $(0,79-0,92)$ \\
Razón inicio actividad sexual (bajo coerción / bajo efectos del alcohol) & 0,013 & 2,01 & $(1,15-3,50)$ \\
\hline
\end{tabular}

Tabla V

\section{AJUSTE DE MODELO REGRESIÓN LOGÍSTICA PARA VARIABLES SOCIODEMOGRÁFICAS Y FAMILIARES Y ANTECEDENTE DE AGRESIÓN SEXUAL}

\begin{tabular}{lccc}
\hline Variables & Valor $\mathrm{p}$ & OR & IC 95\% \\
\hline Relaciones familiares (mala) & 0,000 & 2,20 & $(1,52-3,17)$ \\
Relaciones familiares (regular) & 0,001 & 1,48 & $(1,19-1,85)$ \\
Tipo de permiso semana (sin restricción) & 0,036 & 2,01 & $(1,04-3,86)$ \\
Tipo de permisos fin semana (sin restricción) & 0,019 & 1,30 & $(1,04-1,61)$ \\
Actividad padre (trabajo no calificado) & 0,035 & 1,29 & $(1,02-1,65)$ \\
Escolaridad & 0,028 & 0,92 & $(0,85-0,99)$ \\
Escolaridad madre & 0,011 & 0,96 & $(0,93-0,99)$ \\
Con quien conversa de sexualidad (nadie) & 0,012 & 1,92 & $(1,15-3,18)$ \\
Supervisión (otros) & 0,031 & 1,51 & $(1,04-2,21)$ \\
\hline
\end{tabular}

Concordante también con la literatura $(31,32)$, este estudio mostró asociación entre la agresión sexual y consumo de alcohol, consumo de drogas e inicio precoz del consumo de drogas. Pero además mostró asociación entre agresión sexual y consumo de tabaco, lo que no ha sido reportado en otros estudios $(31,32)$.

Debido a que el promedio de edad a la agresión fue 10,3 años, y el promedio de edad de inicio del consumo de tabaco, alcohol y drogas, y actividad sexual fue posterior (14 y 15 años), estos comportamientos pueden estar asociados directamente al abuso sexual.

Concordante con la literatura (33), este estudio también mostró asociación entre factores familiares adversos como malas relaciones entre padres e hija, deficiente comunicación entre padres e hija, especialmente en los temas de sexualidad, inestabilidad familiar, no crianza por ambos padres, deficiente supervisión parental, y el antecedente de agresión sexual. No está claro si estos antecedentes familiares adversos estaban presentes antes que el evento de la agresión sexual ocurriera.

Este estudio muestra un alto porcentaje $(22,7 \%)$ de antecedente de agresión sexual en adolescentes de nivel socioeconómico medio-bajo de sectores urbanos, que consultaron en un centro especializado en la atención de salud sexual y reproductiva para adolescentes. Este antecedente se asocia a conductas que aumentan el riesgo de enfermedades, afecta los vínculos de pareja y aumenta el consumo de substancias.

Es esencial que los profesionales de salud aprendan a identificar conductas de riesgo que llevan a la sospecha de agresión sexual, a la vez que estén capacitados para intervenir apropiadamente a las pacientes que develen el antecedente. Estas conductas se pueden extender más allá de la etapa de la adolescencia. Los esfuerzos de las políticas públicas debieran principalmente estar orientados a fortalecer programas de prevención primaria del abuso sexual, lo que contribuiría a disminuir las traumáticas y costosas consecuencias para las víctimas. 
Este estudio tiene limitaciones ya que sus resultados pueden no ser aplicables a la población adolescente femenina de otros estratos socioeconómicos o de sectores rurales.

\section{CONCLUSIÓN}

El antecedente de agresión sexual en adolescentes mujeres se asocia a conductas tales como inicio sexual temprano, actividad sexual no protegida, mayor número de parejas sexuales, embarazos producto de violación, e ITS. Los profesionales de salud, en especial, salud sexual y reproductiva, debieran estar capacitados para detectar este antecedente y así intervenir en forma adecuada y eficaz.

\section{REFERENCIAS}

1. López F, Carpintero E, Hernandez A, Marin MJ, Fuertes $A$. Prevalence and sequelae of childhood sexual abuse in Spain. Child Abuse Negl 1995;19:1094-50.

2. Browne A, Finkelhor D. Impact of child sexual abuse: A review of the research. Psychological Bulletin 1986;99:66-77.

3. Cahill C, Llewelyn SP, Pearson C. Long-term effects of sexual abuse which occurred in childhood: A review. Br J Clin Psychology 1991;30:117-30.

4. Finkelhor D, Browne A. The traumatic impact of child sexual abuse: A conceptualization. Am J Orthopsychiatry $1985 ; 55: 530-41$

5. Gómez P, Molina R, Zamberlin N. Factores relacionados con el embarazo y la maternidad en menores de 15 años en América Latina y el Caribe. Doc 1 Promsex/Flasog, enero 2011, Lima Perú. Disponible en: http://www.spog.org.pe/web/images/spog/DOCs/ Documentos_Tecnicos/FactoresRelacionadosEmbarazoMenos15anosAL.pdf.

6. Nahuelpán E, Varas J. La violencia de género en Chile, período 2000-2010. Una reflexión a partir del análisis de las agresiones sexuales constatados en el Servicio Médico Legal. Abril 2011. Disponible en: http://www.sml.cl/proyectos/estadistica/documentos/ VIOLENCIA_GENERO_2000-2010.pdf.

7. González E, Montero A, Martínez V, Leyton C, Luttges C, Molina T. Características y consecuencias de las agresiones sexuales en adolescentes consultantes en un centro de salud sexual y reproductiva. Rev Chil Obstet Ginecol 2012;77:413-22.

8. Naciones Unidas. Informe de la Conferencia Internacional sobre la Población y el Desarrollo. El Cairo, 1994. Disponible en: http://www.unfpa.org/webdav/ site/global/shared/documents/publications/2004/icpd spa.pdf.

9. Fergusson DM, Lynskey MT, Horwood LJ . Childhood sexual abuse and psychiatric disorder in young adulthood: I. Prevalence of sexual abuse and factors associated with sexual abuse. J Am Acad Child Adolesc Psychiatry 1996;35:1355-64.

10. Pereda N. Consecuencias psicológicas a largo plazo del abuso sexual infantil. Papeles del Psicólogo;2010,3(2):191-201. Disponible en: http://www.papelesdelpsicologo.es/pdf/1846.pdf.

11. Leserman J. Sexual abuse history: Prevalence, health effects, mediators, and psychological treatment. Psy- chosomatic Med 2005;67:906-15.

12. Kendall-Tackett AK, Meyer Williams L, Finkelhor D. Impact of sexual abuse on children: A review and synthesis of recent empirical studies, Psyhological Bulletin 1993;113:164-80.

13. Consertino C, Meyer-Bahlburg H, Albert JL, Weinberg SL. Sexual behavior problems and psychopathology symptoms in sexually abused girls. J Am Acad Child Adolesc Psychiatry 1995;43:1033-42.

14. Fajewonyomi BA, Orji EO, Adeyemo AO. Sexual dysfunction among female patients of reproductive age in a hospital setting in Nigeria. J Health Popul Nutr 2007;25:101-6.

15. Finkelhor D, Hotaling G Lewis I, Smith C. Sexual abuse and its relationship to later sexual satisfaction, marital status, religion and attitudes. J Interpersonal Violence 1993;4:279-99.

16. Brown NL, Wilson SR, Kao Ya-Min, Luna V, Kuo ES, Rodriguez C, Lavori WP. Correlates of sexual abuse and subsequent risk taking. Hispanic $\mathrm{J}$ Behavioral Sciences 2003;25:331-51.

17. Buzi RS, Tortolero SR, Roberts RE, Ross MW, Addy $\mathrm{RC}$, Christine M Markham. The impact of a history of sexual abuse on high-risk sexual behaviors among females attending alternative schools. Adolescence 2003;38:595-605.

18. Vitriol GV, Vásquez M, Iturra LI, Muñoz RC, Diagnóstico y abordaje de secuelas por abuso sexual infantil, en tres mujeres consultantes a un servicio de salud mental de hospital general. Rev Chil Neuro-Psiquiat 2007;45:20-8.

19. Senn ET, Carey PM, Vanable AP, Childhood and adolescent sexual abuse and subsequent sexual risk behavior: Evidence from controlled studies, methodological critique, and suggestions for research. Clin Psichol Review 2008;28:711-35.

20. Heisse LL. Reproductive freedom and violence against women: where are the intersections? J Law Med Ethics 1993;21:206-16.

21. Bohn DK, Holz KA. Sequelae of abuse. Health effects of childhood sexual abuse, domestic battering and rape. J Nurse Midwifery 1996;41:442-56.

22. McMahon PM, Goodwin MM, Stringer G. Sexual violence and reproductive health. Matern Child Health $\mathrm{J}$ 2000;4:121-4.

23. Twill SE, Green DM, Traylor A. A descriptive study on sexually exploited children in residential treatment. Child Youth Care Forum 2010;39:187-99.

24. Williams C, Larsen U, McCloskey LA. The impact of childhood sexual abuse and intimate partner violence on sexually transmitted infections. Violence Vict 2010;25:787-98.

25. Khan N. Sexual and physical abuse: a threat to reproductive and sexual health. Sex Health Exch 1998;1:13.

26. Klein H, Chao BS. Sexual abuse during childhood and adolescence as predictors of HIV-Related sexual risk during adulthood among female sexual partners of injection drug users, Violence Against Women 1995;1:55-76.

27. Molina R, González E, Sandoval J. Embarazo en adolescentes producto de una agresión sexual: alto riesgo obstétrico y perinatal. Rev Sogia 1995; 2:74-82.

28. Boyer D, Fine D. Sexual abuse as a factor in adolescent pregnancy and child maltreatment. Fam Plann Perspect 1992;24:4-11. 
29. Roosa MW, Tein JY, Reinholtz C, Angelini PJ. The relationship of childhood sexual abuse to teenage pregnancy. J Marriage Fam 1997;59:119-30.

30. Stock J, Bell M, Boyer D, Connell F. Adolescent pregnancy and sexual risk-taking among sexually abused girls. Fam Plann Perspect 1997;29:200-27.

31. Ramos-Lira L, Saldívar-Hernández G, Medina-Mora M E, Rojas-Guiot E, Villatoro-Velasquez J. Prevalencia de abuso sexual en estudiantes y su relación con el consumo de drogas. Salud Pública de México 1998;40:221-33.

32. Luster T, Small SA. Sexual abuse history and problems in adolescence: exploring the effects of moderating variables. J Marriage Fam 1997;59:131-42.
33. González E, Molina T, Toledo V, Luengo X, Molina C. Violación intra y extrafamiliar en adolescentes: variables personales y familiares seleccionadas. Rev Sogia 1997;4:13-21.

34. Fergusson D, Horwood J, Lynskey M. Childhood sexual abuse, adolescent sexual behaviors and sexual revictimization. Child Abuse Negl 1997;21:789803.

35. Código Penal de la República de Chile. Ley №19.617 y No19.927.

36. Hosmer DW, Lemeshow S. Applied Logistic Regression, 2nd Edition, Ed. New York:John Wiley \& Sons. INC. 2000. 